\title{
CMD in Antwerp
}

A report compiled by Europhysics News from the notes provided by $\mathbf{H}$. Bilz, V. Heine, W. Kohn, A. Libchaber, G. Toulouse, and the closing address of the Conference Chairman, J.T. Devreese.

The 1980 Annual Conference of the EPS Condensed Matter Division was not only the latest in a series but in real terms a new beginning. (Heine) With nearly 600 participants, over 60 invited speakers and 350 contributed papers, Antwerp marked a big step forward in European collaboration in condensed matter physics. Many people had voiced the need for a general European conference covering all aspects of the field, similar to the annual "March Meeting" of the American Physical Society. The Antwerp conference was the first conference of comparable scope and with a wide enough participation for it to be genuinely European. It was widely acclaimed as a great success for EPS, as well as for Professor Balkanski, outgoing chairman of the CM Division and his successor and Chairman of the Conference, Professor Devreese, whose personal initiative and energy showed that such a conference is feasible in the European context.

Condensed matter physics has a problem. It is not a branch of physics but a whole constellation of subjects: from magnetism to lattice dynamics, from alloys to semiconductor surfaces, phase transitions of diverse types, etc, etc. These are all substantial subjects in themselves, each with a richness of phenomena, of experimental techniques and theoretical aspects. Thus it is easy to understand that small in tensive topical conferences on specialized subjects have been popular in EPS. But all condensed matter physicists should recognize the importance of cross fertilisation of ideas between these various subjects. The boundaries are constantly changing, with growth points of new specialities often arising from the interface between older areas. Where would the study of quasi one- and two-dimensional systems fit in the demarcations of 10 years ago? Should superionic conductors be classified with ionic materials, transport theory or the physics of defects? Moreover, and this is an important point, the techniques of the theoretician often run orthogonal to the techniques of the experimentalist, each cross-fertilising the other. To a theoretician, a disordered medium may equally well represent an alloy, amorphous semiconductor, spin glass, or the spin disorder of a ferromagnet above the Curie temperature. Large general condensed matter conferences with several parallel sessions are a necessary source of stimulation for the lively development of the field.
Support for this principle came from Walter Kohn, seeing the European scene through American eyes. "Without the opportunity to learn about what goes on outside our own specialties we should all soon become pretty stale. For me, at least, it is much easier to learn at such a meeting than by reading the literature, which is much too overwhelming and which does not permit questions. The "feel" of an area of research comes across much better in live, hot presentations than in cold print. I hope very much that similar meetings of this kind will be held on a regular basis in the coming years. They are invaluable for the younger researchers whose interests should be especially kept in mind".

The USA apart, general conferences in condensed matter have been held in the Federal Republic of Germany, The Netherlands, and the U.K. on an annual basis. Now we are ready for a Europe-wide approach, as the poll taken at Antwerp clearly indicated, and we can expect that in future, European efforts will be united under the banner of the EPS.

\section{Condensed Matter Physics}

A general conference is not the easiest of events to report on, but the following com ments should give some idea of the scope of the Conference. The Proceedings, including the invited papers and the Conference Chairman's closing address will be published by Plenum Press; contributed papers will be published in a special issue of Solid State Communications. The Abstracts have appeared as Volume $3 \mathrm{G}$ in the Europhysics Conference Abstracts Series.

\section{Plenary Presentations (Devreese)}

To highlight the originality and quality of European work one could perhaps pick out the papers of Isaac Silvera - The Creation of a New Quantum Gas: Atomic Hydrogen (see Page 9), and Anatole Abragam's review of experiments on Nuclear Antiferromagnetism and Ferromagnetism. So selective, however, had the large international programme committee been, the standard throughout was very high indeed. In the plenary sessions we had also:

- Morrel Cohen's analysis of elementary excitations in topologically disordered materials in which he associated frustration in random magnetic systems with localization (à la Lifshitz) and proposed that two types of bounds to spectra exist: normal band edges connected with extended states, and limits connected with bound states and a depression of the level density which can explain, for example, the sharpness of the edge in amorphous silicon.

- Jim Phillips' topological approach to covalent and metallic glasses. He proposed that the glass transforming tendency is optimized when the number of degrees of freedom equals the number of contraints, the former relating to the entropy, and the latter to the strain field. His ideas are the microscopic analogue of the "entropy crisis" discussed by Kauzman.

- Manuel Cardona's evaluation of the problem of hydrogen in amorphous $\mathrm{Si}$, which is of outstanding practical importance for the possible future development of inexpensive solar cells.

- Volker Heine's attack on the old but unsolved problem of the magnetic state of iron above $T_{C}$ in which he demonstrated the power of modern computational techniques. He writes that the question mirrors a more general problem in phase transitions. In the Stone's theory of the 1930's, all magnetism disappears above $T_{C}$, giving a normal metal, and this is analogous to the current theories of charge density waves (periodic lattice distortions) found in some materials like $\mathrm{Ta} \mathrm{Se}_{2}$. However experiment shows that local lattice distortions exist above $T_{C}$ giving rise to local magnetic moments in iron. It is difficult to measure directly the magnitude of the atomic moment, the degree and nature of short range order, and the fluctuations, so numerical computations have been used to explore the energies of various regular and irregular configurations of spins, with the magnitude of the local moment calculated self-consistently in each case. Just as in the past, band structure calculations stimulated the new ideas of pseudopotentials and of the resonance theory of transition metals, so the present calculations have resulted in new theoretical developments. In a metal like iron we must think of the electrons as delocalised in Bloch-like states. Why do the $10^{23}$ electrons then conspire to give a behaviour that is somewhat similar to localised Heisenberg atomic moments? Following ideas of Pettifor, we have outlined a theoretical proof that the disorderd local moment state has lower energy that the non-magnetic state for any tight binding band, somewhere in the range one-third to two-thirds filled.

- Walter Kohn's generalizations of his density functional method which is one of the powerful tools in present day theoretical solid state physics. The nature of the gradient expansion however, still remains an interesting question (is it convergent, asymptotic, ...?). 
- Heinz Bilz'analysis of nonlinear excitations in ferroelectrics. He writes that his approach is based on the observation of a strong repulsive electron-two phonon coupling in ferroelectric oxides and chalcogenides. The microscopic origin of this nonlinear coupling may be found in the local dynamical instability of the outer $2 p$ electrons of the oxygen ion and similarly for sulphites etc. The model in a selfconsistent phonon approximation, reproduces the temperature-dependence of ferroelectric soft modes. In a pseudo one dimensional version, the model exhibits exact non-linear solutions. The static excitations are kinks (domains) and di-polar flips similar to a phi 4 model. In addition, exact periodic solutions of coupled polaron plasmon waves (periodons) have been found. They are reminiscent of inhomogeneous photo-induced phenomena in ferroelectrics. Moreover, it seems that the model may be applicable to biological systems.

\section{Symposia}

In the Symposia, from the many significant contributions presented, the following points have been abstracted as being amongst the most stimulating, at least to a theoretician (Devreese).

For strongly disordered threedimensional systems we heard about an explicit method to derive localization, and also of experimental work which suggests that there is a relation to Wigner localization in two-dimensional systems. A more profound insight into the Wigner localization (e.g. the theoretical determination of the electron density at which it occurs) will require further studies of the electron gas beyond the random phase approximation (e.g. including dynamical exchange).

The observation of superconductivity in a truly organic one-dimensional material belonging to the TCNO family and labelled (HMTSF) ${ }_{2} \mathrm{PF}_{6}$ is a most recent and exciting discovery. This will certainly lead to an intensification of studies on onedimensional materials and stimulate efforts to synthesize new materials by ingenious chemical engineering.

In the session on phase changes, we heard about probing time-dependent phenomena on a macroscopic scale by means of neutron scattering, and also of X-ray diffraction and NMR studies of lyotropic liquid crystals (such as soap). These last which indicate that lyotropic structures can be described as ordered entanglements of two liquids, are relevant to our understanding biological membranes.

It is clear that we are going to hear much more about polarons in the future. They have been described as electrons that cannot "streak", while more formally they are conduction electrons which create a distortion of the surrounding ionic or polar lat- tice; their properties are quite distinctive. The Conference was given a comprehensive account of Fred Brown's beautiful experimental work and also the results of the most recent Japanese measurements on polaron mobility and their streaming motion.

In the field of dielectric properties, one could note the inspired choice of analysing diverse phenomena in terms of a universal mechanism based on the density of states as being linear in energy and leading to a generalized understanding of many low frequency characteristics of solids. Attention was also drawn to the continuing study of the A15 compounds in general and the possible role of acoustical surface plasmons in superconductivity. The question then arises as to the stability of these acoustical plasmons.

Correlation effects and local fields in energy band theory of insulators and semiconductors together with the important problem of a first principle phonon calculation for covalent semiconductors constitute a vital field of great complexity and significant recent progress. The Stieltjes moment expansion of the polarizability together with the consistency between the electronion potential in the dynamical matrix and the pseudopotential used in the bandstructure calculation have been shown to be crucial here.

The study of defects seems to have shifted from fundamental defects in simple solids to extended complexes in more complicated solids.

Outstanding results were communicated to the Conference on fluids notably on polarized liquid ${ }^{3} \mathrm{He}$, elementary excitations in liquid ${ }^{3} \mathrm{He}$, as well as work on the onset of turbulence in a Rayleigh-Benard experiment in He. Libchaber notes that, for a fluid of small Prandtl number, in a box containing two convective rolls, a chaotic state can be reached after the destabilization of only two oscillators. The cascade of bifurcations leading to turbulence follows the route thus: above the onset of convection, the next bifurcation leads to an oscillator associated to a transverse vibration of the convective rolls. A second oscillator is destabilized for a larger value of the temperature difference, followed by a lock-in state between the two oscillators. Just before the onset of turbulence a cascade of frequency demultiplication (period doubling) of the lowest frequency finally generates a low frequency noise.

In the field of surface physics one sees that an understanding of the detailed mechanism of molecular and atomic adsorption and of the structure of molecules at surfaces is developing. Semiconductors are a major preoccupation and the field is in continous evolution. The symposium devoted to this subject was characterized by first attempts to investigate the transport in semiconductor devices with submi- cron geometries. Our present understanding of narrow gap and semi-magnetic semiconductors was critically reviewed along with screening effects due to plasmons and polaritions.

A complete understanding of spin glasses is still an open problem of statistical mechanics, but in the words of Toulouse, one of our major concerns is the construction of a mean field theory, as in working through the infinite-ranged model proposed by $D$. Sherrington and S. Kirkpatrick theoreticians have stumbled at least four times on unexpected rocks.

- First surprise: the free energy of the spin glass phase was found to lie higher in energy than the analytic continuation of the free energy of the paramagnetic phase. - Second surprise: in the use of the "replica trick" unacceptable results, such as a negative entropy, were obtained unless the replica symmetry was spontaneously broken.

- Third surprise: it was found that in the $(H, T)$ phase diagram, the spin glass transition temperature on the zero-field axis was not an isolated point, contrary to all expectations, but the end-point of a transition line (in a way somewhat reminiscent of antiferromagnets).

- Last but not least, both from internal consistency arguments and from MonteCarlo simulations, arose the evidence that linear response theory was violated in the spin glass phase.

What the relevance of all these theoretical mysteries is to the materials which are actively investigated by the experimentalists, is a question open to debate.

Angular resolved photoemission spectroscopy is a technique of growing importance and spin polarized photoemission studies have led to a breakthrough in our understanding of electronic structure in solids. Discrepancies between band theory and experiment (e.g. in $\mathrm{Ni}$, where the first results on spin polarized photoemission were presented) are a stimulus for theorists to undertake detailed studies of dynamical correlations (and exchange) accompanying the excitation of conduction electrons.

\section{Conclusion}

From the wide variety of subjects covered, it is tempting to see if recent solid state physics in Europe has a specific character when compared to developments in the U.S.A. Some have already been mentioned and there are no doubt others such as EXAFS and superlattices. We saw however, many examples of major developments which have taken place at a level of true international collaboration, of which the recent work on localization and resistance in thin wires and films is a nice example. Condensed matter research is not a regional affair but one of world wide significance. 